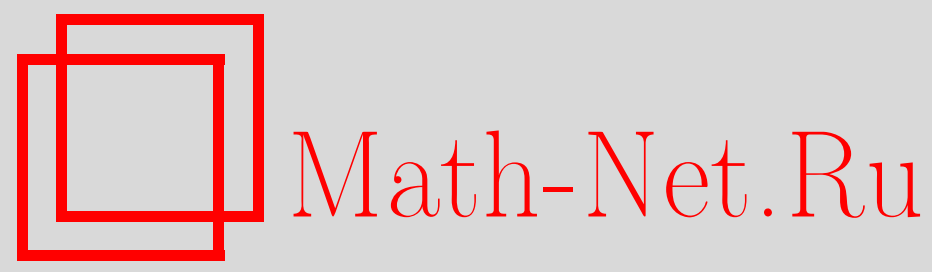

А. В. Тарасов, Обобщение критерия биюнктивности Шефера, Дискрет. матем., 2012, том 24, выпуск 2, 92-99

DOI: https://doi.org/10.4213/dm1186

Использование Общероссийского математического портала Math-Net.Ru подразумевает, что вы прочитали и согласны с пользовательским соглашением http://www.mathnet.ru/rus/agreement

Параметры загрузки:

IP: 3.91 .87 .62

26 апреля 2023 г., $17: 24: 16$ 


\title{
Обобщение критерия биюнктивности Шефера
}

\author{
(c) 2012 г. A. В. Тарасов
}

\begin{abstract}
Критерий биюнктивности Шефера состоит в том, что булева функция $f$ биюнктивна, то есть представима в виде 2-КНФ, тогда и только тогда, когда ее множество выполняющих векторов $E_{f}$ замкнуто относительно операции, задаваемой покоординатным применением функции голосования от трех переменных. В данной работе приведено обобщение данного критерия на случай, когда множество $E_{f}$ замкнуто относительно операции, задаваемой покоординатным применением пороговой функции из более широкого класса.
\end{abstract}

Введем следующие обозначения: пусть $V_{n}$ есть множество двоичных векторов длины $n$; $1_{n}$ есть вектор из $V_{n}$, состоящий из единиц; если $\alpha \in V_{n}$, то через $\alpha(i)$ обозначим значение $i$-й координаты вектора $\alpha, i=1,2, \ldots, n$; и пусть $f_{i_{1} \ldots i_{k}}^{a_{1} \ldots a_{k}}\left(x_{1}, \ldots, x_{n}\right)$ - подфункция булевой функции $f\left(x_{1}, \ldots, x_{n}\right): V_{n} \rightarrow\{0,1\}$, полученная фиксацией переменных $x_{i_{1}}, \ldots, x_{i_{k}}$ значениями $a_{1}, \ldots, a_{k}, 1 \leqslant k \leqslant n$.

Для всякой булевой функции $f\left(x_{1}, \ldots, x_{n}\right)$ множество

$$
E_{f}=\left\{\alpha=\left(a_{1}, \ldots, a_{n}\right): f\left(a_{1}, \ldots, a_{n}\right)=1\right\}
$$

будем называть множеством выполняющих векторов функции $f$.

Символами $\lfloor\cdot\rfloor$ и $\lceil\cdot\rceil$ обозначим, соответственно, нижние и верхние целые части вещественных чисел.

Булева функция $f\left(x_{1}, \ldots, x_{n}\right)$ называется

биюнктивной, если $f \equiv 1$ или существует представление функции $f$ в виде 2-КНФ:

$$
f\left(x_{1}, \ldots, x_{n}\right) \equiv \bigwedge_{i=1}^{t}\left(x_{s_{i, 1}}^{a_{i, 1}} \vee x_{s_{i, 2}}^{a_{i, 2}}\right), \quad a_{i, 1}, a_{i, 2} \in\{0,1\}, \quad i=1,2, \ldots, t
$$

слабо положительной, если $f \equiv 1$ или существует представление $f$ в виде следующей КНФ:

$$
f\left(x_{1}, \ldots, x_{n}\right) \equiv \bigwedge_{i=1}^{t}\left(x_{s_{i, 1}}^{a_{i}} \vee x_{s_{i, 2}} \vee \ldots \vee x_{s_{i, k_{i}}}\right), \quad a_{i} \in\{0,1\}, \quad i=1,2, \ldots, t
$$

слабо отрицательной, если $f \equiv 1$ или существует представление $f$ в виде следующей КНФ:

$$
f\left(x_{1}, \ldots, x_{n}\right) \equiv \bigwedge_{i=1}^{t}\left(x_{s_{i, 1}}^{a_{i}} \vee \bar{x}_{s_{i, 2}} \vee \ldots \vee \bar{x}_{s_{i, k_{i}}}\right), \quad a_{i} \in\{0,1\}, \quad i=1,2, \ldots, t .
$$


Множества всех функций из этих классов обозначим $\mathrm{Bi}, \mathrm{WP}, \mathrm{WN}$ соответственно. Формулы (1)-(3) для классов функций Bi, WP, WN будем называть приведенными представлениями. В [2-4] показано, что классы Bi, WP, WN, наряду с классом функций, представимых в виде произведения аффинных функций, порождают полиномиально решаемые классы систем уравнений без ограничений на выбор неизвестных и с ограничениями на выбор функций, исследован ряд их свойств.

Для произвольной булевой функции $f$ элементарную дизъюнкцию $\varphi$, удовлетворяющую условию $f \cdot \varphi \equiv f$, будем называть имплицентой функции $f$, а если для любой ее части $\varphi^{\prime}$ условие $f \cdot \varphi^{\prime} \equiv f$ не выполнено, то такую имплиценту назовем простой.

Длиной элементарной дизъюнкции назовем число входящих в нее переменных. Подкласс класса слабо положительных функций, в приведенном представлении которых вида (2) имплиценты, содержащие переменную с отрицанием, имеют длину не более $k$ (то есть $\left.k_{i} \leqslant k\right)$, а остальные - длину не более $r$, обозначим через $\mathrm{WP}_{r, k}$. Аналогичным образом определяется подкласс $\mathrm{WN}_{r, k}$ класса слабо отрицательных функций, в приведенном представлении которых вида (3) имплиценты, содержащие переменную без отрицания, имеют длину не более $k$, а остальные имплиценты - длину не более $r$. В дальнейшем будем предполагать, что имплиценты в приведенных представлениях (1)-(3) являются простыми, то есть их собственные подформулы не являются имплицентами рассматриваемых функций.

Пусть $r \geqslant 2,1 \leqslant k \leqslant r$. Определим пороговую функцию $v_{r, k}$ по правилу

$$
v_{r, k}\left(x_{1}, x_{2}, \ldots, x_{r}\right)= \begin{cases}1, & \text { если } x_{1}+x_{2}+\ldots+x_{r} \geqslant k, \\ 0, & \text { если } x_{1}+x_{2}+\ldots+x_{r}<k\end{cases}
$$

Легко видеть, что

$$
v_{r, k}\left(x_{1}, x_{2}, \ldots, x_{r}\right)=\bigvee_{1 \leqslant i_{1}<\ldots<i_{k} \leqslant r} x_{i_{1}} \cdots x_{i_{k}},
$$

в частности,

$$
v_{3,2}\left(x_{1}, x_{2}, x_{3}\right)=x_{1} x_{2} \vee x_{1} x_{3} \vee x_{2} x_{3} .
$$

Определим функцию

$$
w_{r, k}=v_{r, k} \oplus 1,
$$

которая, исходя из (5), может быть задана формулой:

$$
w_{r, k}\left(x_{1}, x_{2}, \ldots, x_{r}\right)=\bigwedge_{1 \leqslant i_{1}<\ldots<i_{k} \leqslant r}\left(\bar{x}_{i_{1}} \vee \ldots \vee \bar{x}_{i_{k}}\right) .
$$

Определим $r$-арные операции $\tilde{v}_{r, k}$ и $\widetilde{w}_{r, k}$ на множестве $V_{n}$ как операции покоординатного применения функций, задаваемых равенствами (5) и (6).

Множество $M \subset V_{n}$ будем называть $\tilde{v}_{r, k}$-замкнутым ( $\tilde{w}_{r, k}$-замкнутым), если для любых $\alpha_{1}, \ldots, \alpha_{r} \in M$ вектор $\tilde{v}_{r, k}\left(\alpha_{1}, \ldots, \alpha_{r}\right) \in M$ (соответственно $\left.\tilde{w}_{r, k}\left(\alpha_{1}, \ldots, \alpha_{r}\right) \in M\right)$.

В работах Т. Шефера [1] и С. П. Горшкова [2] доказан критерий биюнктивности, который во введенных обозначениях можно сформулировать следующим образом.

Теорема 1. Булева функиия $f\left(x_{1}, \ldots, x_{n}\right)$ биюнктивна тогда и только тогда, когда множество $E_{f}$ является $\tilde{v}_{3,2}$-замкнутылм. 
Докажем обобщение данной теоремы. Сначала напомним некоторые понятия, сформулируем и докажем промежуточные результаты.

Если $f\left(x_{1}, \ldots, x_{n}\right)$ - булева функция, то обозначим через $[f]$ класс функций, представимых формулами над функцией $f$. В данный класс будут входить функции, получаемые из функции $f$ с помощью операций отождествления переменных и суперпозиций над функцией $f$ (см. [5, 6]).

Лемма 1. Пусть $r \geqslant 3,2 \leqslant k \leqslant r-1, m=\lceil r / k-1\rceil$. Тогда $v_{m, 2} \in\left[v_{r, k}\right], w_{m, 2} \in\left[w_{r, k}\right]$.

Доказательство. По условию леммы, $r=m(k-1)+s$, где $0 \leqslant s<k-1$. Рассмотрим функцию

$$
f\left(x_{1}, \ldots, x_{m}\right)=v_{r, k}(\underbrace{x_{1}, \ldots, x_{1}}_{k-1}, \underbrace{x_{2}, \ldots, x_{2}}_{k-1}, \ldots, \underbrace{x_{m-1}, \ldots, x_{m-1}}_{k-1}, \underbrace{x_{m}, \ldots, x_{m}}_{s}) .
$$

Воспользуемся представлением (5) для функции $v_{r, k}$ и выпишем ДНФ функции $f$. Очевидно, что данная ДНФ будет содержать все $\left(\begin{array}{c}m \\ 2\end{array}\right)$ элементарных коньюнкций вида $x_{i} x_{j}$, $1 \leqslant i<j \leqslant m$, и не будет содержать элементарных конъюнкций вида $x_{i}, 1 \leqslant i \leqslant m$. Далее, все остальные элементарные конъюнкции, получаемые из (5) путем отождествления переменных в соответствии с (7), будут, очевидно, поглощаться элементарными конъюнкциями длины 2. Поэтому

$$
f\left(x_{1}, \ldots, x_{m}\right)=v_{m, 2}\left(x_{1}, \ldots, x_{m}\right) .
$$

Аналогично проводится доказательство для функций $w_{m, 2}, w_{r, k}$. Лемма доказана.

Следствием данной леммы является следующее утверждение.

Следствие 1. В условиях леммы 1 , из $\tilde{v}_{r, k}$-замкнутости множества $M \subset V_{n}$ следует его

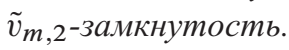

Доказательство. Если $\alpha_{1}, \ldots, \alpha_{m} \in M$, то

$$
\tilde{v}_{m, 2}\left(\alpha_{1}, \ldots, \alpha_{m}\right)=\tilde{v}_{r, k}(\underbrace{\alpha_{1}, \ldots, \alpha_{1}}_{k-1}, \underbrace{\alpha_{2}, \ldots, \alpha_{2}}_{k-1}, \ldots, \underbrace{\alpha_{m-1}, \ldots, \alpha_{m-1}}_{k-1}, \underbrace{\alpha_{m}, \ldots, \alpha_{m}}_{s}) \in M,
$$

Следствие доказано.

Заметим, что функция $v_{3,2}$ является монотонной и самодвойственной, порождая замкнутый класс SM самодвойственных монотонных функций (см. [6]). На основе этого факта можно получить следующее обобщение теоремы 1.

Теорема 2. Функиия $f$ биюнктивна тогда и только тогда, когда множество $E_{f}$ замкнуто относительно покоординатного применения любой монотонной самодвойственной функциии.

Доказательство. Если $f$ биюнктивна, то множество $E_{f}$ является $u$-замкнутым для любой монотонной самодвойственной функции $u$, поскольку она лежит в классе $\left[v_{3,2}\right]$.

Пусть теперь $u \in\left[v_{3,2}\right]$ и множество $E_{f} u$-замкнуто. Следуя обозначениям книги [6], можно говорить о том, что замкнутый класс $\left[v_{3,2}\right]$ является подалгеброй алгебры булевых функций с операциями циклической перестановки, транспозиции, отождествления двух первых переменных, введения фиктивной переменной и операцией суперпозиции вида

$$
(h * g)\left(x_{1}, \ldots, x_{m}, x_{m+1}, \ldots, x_{m+n-1}\right)=h\left(g\left(x_{1}, \ldots, x_{m}\right), x_{m+1}, \ldots, x_{m+n-1}\right),
$$


где $h\left(x_{1}, \ldots, x_{n}\right), g\left(x_{1}, \ldots, x_{m}\right)-$ функции из класса $\left[v_{3,2}\right]$.

Поскольку функция $v_{3,2}$ симметрическая, нет необходимости рассматривать применение операций циклического сдвига и транспозиции переменных. Аналогично, поскольку

$$
v_{3,2}\left(x_{1}, x_{1}, x_{2}\right)=x_{1}
$$

можно не рассматривать операцию отождествления двух первых переменных.

Поэтому для доказательства теоремы достаточно рассмотреть случай, когда функция $u$ получена из функции $v_{3,2}$ применением только одной из указанных операций, поскольку дальнейшее доказательство очевидным образом получается применением метода математической индукции.

Рассмотрим функцию

$$
\begin{aligned}
u & =\left(v_{3,2} * v_{3,2}\right)\left(x_{1}, x_{2}, x_{3}, x_{4}, x_{5}\right) \\
& =v_{3,2}\left(v_{3,2}\left(x_{1}, x_{2}, x_{3}\right), x_{4}, x_{5}\right) \\
& =x_{1} x_{2} x_{4} \vee x_{1} x_{3} x_{4} \vee x_{2} x_{3} x_{4} \vee x_{1} x_{2} x_{5} \vee x_{1} x_{3} x_{5} \vee x_{2} x_{3} x_{5} \vee x_{4} x_{5} .
\end{aligned}
$$

Легко видеть, что

$$
u\left(x_{1}, x_{2}, x_{3}, x_{1}, x_{2}\right)=v_{3,2}\left(x_{1}, x_{2}, x_{3}\right)
$$

поэтому, если $E_{f} u$-замкнуто, то оно $v_{3,2}$-замкнуто. Теорема доказана.

Таким образом, множество биюнктивных функций совпадает с множеством булевых функций, замкнутых относительно покоординатного применения монотонных самодвойственных функций.

Прежде чем перейти к формулировке и доказательству основного результата данной статьи, сформулируем и докажем следующий несложный факт.

Лемма 2. Для произвольной булевой функиии $f\left(x_{1}, \ldots, x_{n}\right)$ и произвольной элементарной дизъюнкичи

$$
\varphi\left(x_{1}, \ldots, x_{n}\right)=x_{i_{1}}^{a_{1}} \vee \ldots \vee x_{i_{r}}^{a_{k}}
$$

следующие утверждения эквивалентны:

(1) функция ч является простой имплицентой функции $f$;

(2) выполнены условия

$$
f_{i_{1} \ldots i_{k}}^{\bar{a}_{1} \ldots \bar{a}_{k}} \equiv 0
$$

и для всякого $j, 1 \leqslant j \leqslant k$,

$$
f_{i_{1} \ldots i_{j-1} i_{j} i_{j+1} \ldots i_{k}}^{\bar{a}_{1} \ldots \bar{a}_{j-1} a_{j} \bar{a}_{j+1} \ldots \bar{a}_{k}} \not \equiv 0 .
$$

Доказательство. Пусть функция $\varphi$ является простой имплицентой функции $f$, но

$$
f_{i_{1} \ldots i_{j-1} i_{j} i_{j+1} \ldots i_{k}}^{\bar{a}_{1} \ldots \bar{a}_{j-1} a_{j} \bar{a}_{j+1} \ldots \bar{a}_{k}} \equiv 0
$$

для некоторого $j, 1 \leqslant j \leqslant k$. Поскольку

$$
f_{i_{1} \ldots i_{k}}^{\bar{a}_{1} \ldots \bar{a}_{k}} \equiv 0
$$


верно, что

$$
f_{i_{1} \ldots i_{j-1} i_{j+1} \ldots i_{k}}^{\bar{a}_{1} \ldots \bar{a}_{j-1} \bar{a}_{j+1} \ldots \bar{a}_{k}}=x_{i_{j}}^{a_{j}} \cdot f_{i_{1} \ldots i_{j-1} i_{j} i_{j+1} \ldots i_{k}}^{\bar{a}_{1} \ldots \bar{a}_{j-1} a_{j} \bar{a}_{j+1} \ldots \bar{a}_{k}} \vee x_{i_{j}}^{\bar{a}_{j}} \cdot f_{i_{1} \ldots i_{j-1} i_{j} i_{j+1} \ldots i_{k}}^{\bar{a}_{1} \ldots \bar{a}_{j-1} \bar{a}_{j} \bar{a}_{j+1} \ldots \bar{a}_{k}} \equiv 0 .
$$

Тогда элементарная дизъюнкция

$$
\varphi^{\prime}\left(x_{1}, \ldots, x_{n}\right)=x_{i_{1}}^{a_{1}} \vee \ldots \vee x_{i_{j-1}}^{a_{j-1}} \vee x_{i_{j+1}}^{a_{j+1}} \ldots \vee x_{i_{k}}^{a_{k}},
$$

являющаяся частью имплиценты $\varphi$, является, очевидно, имплицентой $f$. Пусть теперь выполнено условие 2 леммы. Поскольку

$$
f_{i_{1} \ldots i_{k}}^{\bar{a}_{1} \ldots \bar{a}_{k}} \equiv 0,
$$

очевидно, что $\varphi-$ имплицента $f$. Если эта имплицента не простая, то найдется ее часть

$$
\varphi^{\prime}\left(x_{1}, \ldots, x_{n}\right)=x_{i_{1}}^{a_{1}} \vee \ldots \vee x_{i_{j-1}}^{a_{j-1}} \vee x_{i_{j+1}}^{a_{j+1}} \ldots \vee x_{i_{k}}^{a_{k}},
$$

являющаяся имплицентой.

В силу разложения

$$
f_{i_{1} \ldots i_{j-1} i_{j+1} \ldots i_{k}}^{\bar{a}_{1} \ldots \bar{a}_{j-1} \bar{a}_{j+1} \ldots \bar{a}_{k}}=x_{i_{j}}^{a_{j}} \cdot f_{i_{1} \ldots i_{j-1} i_{j} i_{j+1} \ldots i_{k}}^{\bar{a}_{1} \ldots \bar{a}_{j-1} a_{j} \bar{a}_{j+1} \ldots \bar{a}_{k}} \vee x_{i_{j}}^{\bar{a}_{j}} \cdot f_{i_{1} \ldots i_{j-1} i_{j} i_{j+1} \ldots i_{k}}^{\bar{a}_{1} \ldots \bar{a}_{j-1} \bar{a}_{j} \bar{a}_{j+1} \ldots \bar{a}_{k}} \equiv 0,
$$

получаем, что

$$
f_{i_{1} \ldots i_{j-1} i_{j} i_{j+1} \ldots i_{k}}^{\bar{a}_{1} \ldots \bar{a}_{j-1} a_{j} \bar{a}_{j+1} \ldots \bar{a}_{k}} \equiv f_{i_{1} \ldots i_{j-1} i_{j} i_{j+1} \ldots i_{k}}^{\bar{a}_{1} \ldots \bar{a}_{j-1} \bar{a}_{j} \bar{a}_{j+1} \ldots \bar{a}_{k}} \equiv 0,
$$

что невозможно. Лемма доказана.

Теорема 3. Пусть $f\left(x_{1}, \ldots, x_{n}\right)$ - булева функиия,

$$
m=\left\lceil\frac{r}{k-1}\right\rceil-1, \quad 2 \leqslant k\left\lfloor\frac{r}{2}\right\rfloor, \quad r \geqslant 4 .
$$

Если множество $E_{f}$ является $\tilde{v}_{r, k}$-замкнутылм, то $f\left(x_{1}, \ldots, x_{n}\right) \in \mathrm{WP}_{m, 2}$. При $k=2$ данное условие является достаточным: множество $E_{f}$ является $\tilde{v}_{r, 2}$-замкнутым тогда и только тогда, когда $f\left(x_{1}, \ldots, x_{n}\right) \in \mathrm{WP}_{r-1,2}$.

Доказательство. Докажем необходимость. В общем случае $k$ - произвольное число, удовлетворяющее неравенствам $2 \leqslant k \leqslant\lfloor r / 2\rfloor$, и для функции $f\left(x_{1}, \ldots, x_{n}\right)$ выполняется следующее свойство: если $\alpha_{1}, \ldots, \alpha_{r} \subset E_{f}$, то $\tilde{v}_{r, k}\left(\alpha_{1}, \ldots, \alpha_{r}\right) \in E_{f}$.

Поскольку $k \leqslant\lfloor r / 2\rfloor$, то $k \leqslant r-k$ и для любых векторов $\alpha_{1}, \alpha_{2} \in E_{f}$ верно включение

$$
\tilde{v}_{r, k}(\underbrace{\alpha_{1}, \ldots, \alpha_{1}}_{k}, \alpha_{2}, \ldots, \alpha_{2})=\alpha_{1} \vee \alpha_{2} \in E_{f},
$$

из которого, по критерию слабой положительности [2], заключающемся в том, что функция $f$ слабо положительна тогда и только тогда, когда множество $E_{f}$ замкнуто относительно операции покоординатной дизъюнкции, следует, что $f \in \mathrm{WP}$.

Теперь воспользуемся доказанным выше следствием 1 . Так как множество $E_{f}$ является $\tilde{v}_{r, k}$-замкнутым, то оно является $\tilde{v}_{m+1,2}$-замкнутым, $m=\lceil r /(k-1)\rceil-1$. Покажем, что в этом случае $f \in \mathrm{WP}_{m, 2}$.

Поскольку $f \in \mathrm{WP}$, существует приведенное представление вида (2), в котором все имплиценты являются простыми. Имплиценты функции $f$ такого приведенного представления (2) имеют вид либо $D=x_{1} \vee \ldots \vee x_{q}$, либо $D=\bar{x}_{1} \vee x_{2} \vee \ldots \vee x_{q}, 1 \leqslant q \leqslant n$. 
Рассмотрим первый случай

$$
D=x_{1} \vee \ldots \vee x_{q}
$$

По лемме 2, для всякого $i, 1 \leqslant i \leqslant q$, выполнено условие $f_{1}^{0 \ldots i} \ldots q .00$. Поэтому для функции $f$ найдутся $q$ выполняющих векторов вида

$$
\begin{aligned}
\alpha_{1} & =\left(1,0, \ldots, 0,0, \alpha_{1}(q+1), \ldots, \alpha_{1}(n)\right), \\
\alpha_{2} & =\left(0,1, \ldots, 0,0, \alpha_{2}(q+1), \ldots, \alpha_{2}(n)\right), \\
& \ldots \\
\alpha_{q} & =\left(0,0, \ldots, 0,1, \alpha_{q}(q+1), \ldots, \alpha_{q}(n)\right) .
\end{aligned}
$$

Легко видеть, что при $q \geqslant m+1$ применение операции $v_{m+1,2}$ к любым $m+1$ векторам из данной совокупности даст вектор

$$
\delta=(\underbrace{0,0, \ldots, 0,0}_{q}, \delta(q+1), \ldots, \delta(n)) \notin E_{D} .
$$

Получаем противоречие с предположением о том, что $q \geqslant m+1$. Следовательно, $q \leqslant m$.

Рассмотрим случай простой имплиценты

$$
D=\bar{x}_{1} \vee x_{2} \vee \ldots \vee x_{q} .
$$

Покажем, что в этом случае $q \leqslant 2$. Аналогично вышесказанному, построим $q$ выполняющих векторов:

$$
\begin{aligned}
\alpha_{1} & =\left(0,0, \ldots, 0,0, \alpha_{1}(q+1), \ldots, \alpha_{1}(n)\right), \\
\alpha_{2} & =\left(1,1, \ldots, 0,0, \alpha_{2}(q+1), \ldots, \alpha_{2}(n)\right), \\
& \ldots \\
\alpha_{q} & =\left(1,0, \ldots, 0,1, \alpha_{q}(q+1), \ldots, \alpha_{q}(n)\right) .
\end{aligned}
$$

При $3 \leqslant q \leqslant m+1$ получаем, что вектор

$$
\begin{aligned}
\delta & =\tilde{v}_{m+1,2}\left(\alpha_{1}, \alpha_{2}, \alpha_{3}, \ldots, \alpha_{q}, \alpha_{1}, \ldots, \alpha_{1}\right) \\
& =(1,0, \ldots, 0, \delta(q+1), \ldots, \delta(n)) \notin E_{f} .
\end{aligned}
$$

Пусть теперь $q>r$. Тогда выберем вектор

$$
\begin{aligned}
\delta & =\tilde{v}_{m+1,2}\left(\alpha_{1}, \alpha_{2}, \alpha_{3}, \ldots, \alpha_{m+1}\right) \\
& =(1,0, \ldots, 0, \delta(q+1), \ldots, \delta(n)) \notin E_{f} .
\end{aligned}
$$

Таким образом, необходимость доказана.

Докажем достаточность. Пусть $k=2$ и $m=\lceil r /(k-1\rceil-1=r-1$. Предположим, что $f\left(x_{1}, \ldots, x_{n}\right) \in \mathrm{WP}_{r-1,2}$ и $\bigwedge_{i=1}^{\mu} D_{i}$ - ее соответствующая приведенная КНФ. Пусть теперь $\left\{\alpha_{1}, \ldots, \alpha_{r}\right\}$ - произвольный набор из $r$ выполняющих векторов функции $f\left(x_{1}, \ldots, x_{n}\right)$. Очевидно, что произвольный вектор из этого набора является выполняющим для элементарной дизъюнкции $D_{i}, i \in\{1, \ldots, \mu\}$. Покажем, что при этом вектор $\delta=\tilde{v}_{r, 2}\left(\alpha_{1}, \ldots, \alpha_{r}\right)$ также является выполняющим и для произвольной элементарной дизъюнкции $D_{i}, i \in\{1, \ldots, \mu\}$. Из этого факта будет следовать, что вектор $\delta$ является 
также выполняющим для функции $f\left(x_{1}, \ldots, x_{n}\right)$. В соответствии с условием теоремы, с точностью до перестановки переменных, элементарные дизъюнкции $D_{i}$ имеют вид либо $D=x_{1} \vee \ldots \vee x_{l}$, где $l \leqslant r-1$, либо $D=\bar{x}_{1} \vee x_{2}$.

Рассмотрим первый случай, когда $D=x_{1} \vee \ldots \vee x_{l}$. Так как $\alpha_{1}, \ldots, \alpha_{r} \in E_{f}$, то $\alpha_{1}, \ldots, \alpha_{r} \in E_{D}$. Значит, каждый из этих векторов содержит по крайней мере по одной единице на местах с 1 -го по $l$-е. Поскольку $l<r$, то найдется координата $i, 1 \leqslant i \leqslant l$, такая, что хотя бы у двух из указанных векторов $i$-я координата равна 1 . В этом случае $i$-я координата вектора $\delta$ равна 1 и $\delta \in E_{D}$.

Пусть теперь $D=\bar{x}_{1} \vee x_{2}$. Поскольку $\alpha_{1}, \ldots, \alpha_{r} \in E_{D}$, то в первых двух координатах этих векторов не может встречаться комбинация $(1,0)$. Если в первых координатах векторов $\alpha_{1}, \ldots, \alpha_{r}$ не более одной единицы, то $\delta(1)=0$ и тогда $\delta \in E_{D}$. Пусть $\delta(1)=1$. Тогда найдутся два вектора $\alpha_{i}, \alpha_{j} \in\left\{\alpha_{1}, \ldots, \alpha_{r}\right\}$ такие, что $\alpha_{i}(1)=\alpha_{j}(1)=1$. Поскольку это выполняющие векторы для $D$, для них выполняются равенства $\alpha_{i}(2)=\alpha_{j}(2)=1$, откуда $\delta(2)=1$ и $\delta \in E_{D}$. Указанные рассуждения верны для любой простой имплиценты $D$ функции $f$.

Теорема доказана.

Результат, аналогичный доказанному в теореме 3 , верен и для пороговой функции $w_{r, k}$.

Теорема 4. Пусть

$$
r \geqslant 4, \quad 2 \leqslant k \leqslant\left\lfloor\frac{r}{2}\right\rfloor, \quad m=\left\lceil\frac{r}{k-1}\right\rceil-1 .
$$

Если множество $E_{f}$ является $\tilde{w}_{r, k}$-замкнутылм, то $f\left(x_{1}, \ldots, x_{n}\right) \in \mathrm{WN}_{m, 2}$. При $k=2$ данное условие является достаточным: множество $E_{f}$ является $\widetilde{w}_{r, 2}$-замкнутым тогда и только тогда, когда $f\left(x_{1}, \ldots, x_{n}\right) \in \mathrm{WN}_{r-1,2}$.

Доказательство. Пусть $f\left(x_{1}, \ldots, x_{n}\right)$ - рассматриваемая булева функция. Положим

$$
f^{\prime}\left(x_{1}, \ldots, x_{n}\right)=f\left(\bar{x}_{1}, \ldots, \bar{x}_{n}\right) \text {. }
$$

Тогда

$$
E_{f^{\prime}}=\left\{\beta=\alpha \oplus 1_{n}: \alpha \in E_{f}\right\}
$$

Поэтому множество $E_{f}$ является $\tilde{w}_{r, k}$-замкнутым тогда и только тогда, когда множество $E_{f^{\prime}}$ является $v_{r, k}$-замкнутым. Теперь остается применить теорему 3.

Резюмируя вышесказанное, можно сделать следующий вывод:

при $r=3, k=2 \tilde{v}_{r, k}$-замкнутость множества $E_{f}$ эквивалентна биюнктивности функции $f$ по критерию Шефера (см. [1]);

при $r \geqslant 4, k=2 \tilde{v}_{r, k}$-замкнутость множества $E_{f}$ эквивалентна принадлежности функции $f$ классу $\mathrm{WP}_{r-1,2}$;

при $r \geqslant 4,3 \leqslant k \leqslant\lfloor r / 2\rfloor$ из $\tilde{v}_{r, k}$-замкнутости множества $E_{f}$ следует принадлежность функции $f$ классу $\mathrm{WP}_{m, 2}, m=\lceil r /(k-1)-1$.

Аналогичным образом формулируются утверждения для $\widetilde{w}_{r, k}$-замкнутости. 


\section{Список литературы}

1. Schaefer T. J., Complexity of satisfiability problems. In: Proc. STOC'78. ACM, New York, 1978, pp. 216-226.

2. Горшков С. П., О сложности распознавания мультиаффинности, биюнктивности, слабой положительности и слабой отрицательности булевых функций. Обозрение прикладной и промышленной математики (1997) 4, №2, 216-237.

3. Горшков С. П., Применение теории $N P$-полных задач для оценки сложности решения систем булевых уравнений. Обозрение прикладной и промышленной математики (1995) 2, №3, 325398.

4. Горшков С. П., О пересечениях классов мультиаффинных, биюнктивных, слабо положительных и слабо отрицательных булевых функций. Обозрение прикладной и промымленной математики (1997) 4, №2, 238-259.

5. Яблонский С. В., Введение в дискретную математику. Наука, Москва, 1979.

6. Марченков С. С., Замкнутые классы булевых функиий. Физматлит, Москва, 2001.

Статья поступила 9.09.2011. 